\title{
Biomimetic Synthesis of Iridoid Alkaloids as Novel Leads for Fungicidal and Insecticidal Agents
}

Qing Xia ${ }^{\dagger *}$, Hao Tian ${ }^{\ddagger}$, Yufei $\mathrm{Li}^{\dagger}$, Xiang $\mathrm{Yu}^{\dagger}$, Weihua Zhang ${ }^{\dagger}$, Qingmin Wang ${ }^{\ddagger *}$

$\dagger$ †iangsu Key Laboratory of Pesticide Science, College of Sciences, Nanjing Agricultural University, Nanjing, 210095, People's Republic of China

\$State Key Laboratory of Elemento-Organic Chemistry, Research Institute of Elemento-Organic Chemistry, College of Chemistry, Collaborative Innovation Center of Chemical Science and Engineering (Tianjin), Nankai University, Tianjin, 300071, People's Republic of China 
Detailed bioassay methods for fungicidal, and insecticidal activities; ${ }^{1} \mathrm{H}$ NMR, ${ }^{13} \mathrm{C}$ NMR and HRMS data of $\mathbf{1}, \mathbf{2}, \mathbf{3 a}-\mathbf{3} \mathbf{c}$, and $\mathbf{3 b} \mathbf{b}-\mathbf{I I}$.

\section{Detailed bioassay procedures for the fungicidal activities}

In Vitro Antifungal Bioassay: The fungicidal activities of compounds were evaluated in mycelial growth tests conducted in artificial media against 14 plant pathogens at a rate of $50 \mu \mathrm{g} / \mathrm{mL}$. Each test compound was dissolved in a suitable amount of acetone and diluted with water containing $0.1 \% \mathrm{TW}-80$ to a concentration of $500 \mu \mathrm{g} / \mathrm{mL}$. To each petri dish was added $1 \mathrm{~mL}$ of the test solution and $9 \mathrm{~mL}$ of culture medium to make a 50 $\mu \mathrm{g} / \mathrm{mL}$ concentration of the test compound, while in another petri dish was added $1 \mathrm{~mL}$ distilled water containing $0.1 \% \mathrm{TW}-80$ and $9 \mathrm{~mL}$ of culture medium as a blank control. A $4 \mathrm{~mm}$ diameter of hyphal growth was cut using a hole puncher on a growing fungal culture and the hyphae were moved to the petri dish containing the test compound. Each assay was performed three times. The dishes were stored in controlled environment cabinets $\left(24 \pm 1{ }^{\circ} \mathrm{C}\right)$ for 4 days, after which the diameter of mycelial growth was measured and the percentage inhibition was calculated using the following equation:

Percentage inhibition $(\%)=($ averaged diameter of mycelia in blank controls - averaged diameter of mycelia in medicated tablets) / (averaged diameter of mycelia in blank controls $-4 \mathrm{~mm}) \times 100$.

\section{Detailed bioassay procedures for the insecticidal activities}

\section{Larvicidal Activities against diamondback moth (Plutella xylostella)}

Stock solutions of each test compound was prepared in dimethylformamide at a concentration of $600 \mu \mathrm{g} / \mathrm{mL}$ and then diluted to the required concentration with water containing TW-20. 
Leaf-dip method was used. Leaf discs $(5 \mathrm{~cm} \times 3 \mathrm{~cm})$ were cut from fresh cabbage leaves (or other leaves) and then dipped into the test solution for $3 \mathrm{~s}$. After air-drying, the treated leaf discs were placed individually into vertical tube (or Petri dishes) and the discs were infested with 10 second-instar diamondback moth larvae. Percentage mortalities were evaluated 3 days after treatment. Evaluations were based on a percentage scale of $0-100$, where 0 equals no activity and 100 equals total kill. Each treatment was performed three times.

\section{Insecticidal Activity against Spider Mite (Tetranychus cinnabarinus Boisduval) Adults}

Sieva bean plants (Phaseolus Vulgaris L.) with primary leaves expanded to $10 \mathrm{~cm}$ were selected and cut back to one plant per pot. A small piece was cut from a leaf taken from the main colony and placed on each leaf of the test plants. This was done about $2 \mathrm{~h}$ before treatment to allow the mites to move to the test plant and to lay eggs. The size of the piece was varied to obtain about 60-100 mites per leaf. At the time of the treatment, the piece of leaf used to transfer the mites was removed and discarded. The mite-infested plants were dipped in the test formulation for $3 \mathrm{~s}$ with agitation and set in the hood to dry. Plants were kept for 2 days before the number of live and dead adults was counted.

\section{Insecticidal Activity against Bean Aphid (Aphis craccivora)}

The insecticidal activities of the compounds were tested against Bean Aphid (Aphis craccivora) by foliar application. About 60 aphids were transferred to the shoot with 3-5 fresh leaves of horsebean. The shoot with aphids was cut and dipped into the solution of $600 \mu \mathrm{g} / \mathrm{mL}$ of test compound for $2 \mathrm{~s}$, after removing extra solutions on the leaf; the aphids were raised in the shoot at $25 \pm 1{ }^{\circ} \mathrm{C}$ and $85 \%$ relative humidity for $16 \mathrm{~h}$. Each experiment for one compound was triplicated. 
methyl

(1R,4aS,7aS)-7-(((tert-butyldimethylsilyl)oxy)methyl)-1-hydroxy-1,4a,5,7a-tetrahydr ocyclopenta[c]pyran-4-carboxylate, 1: ${ }^{1} \mathrm{H}$ NMR $\left(400 \mathrm{MHz}, \mathrm{DMSO}-d_{6}\right) \delta 7.55(\mathrm{~d}, J=$ $6.2 \mathrm{~Hz}, 1 \mathrm{H}), 7.48(\mathrm{~s}, 1 \mathrm{H}), 5.71(\mathrm{~s}, 1 \mathrm{H}), 4.72(\mathrm{t}, J=7.4 \mathrm{~Hz}, 1 \mathrm{H}), 4.39-4.14(\mathrm{~m}, 2 \mathrm{H}), 3.63$ $(\mathrm{s}, 3 \mathrm{H}), 3.01(\mathrm{q}, J=8.3 \mathrm{~Hz}, 1 \mathrm{H}), 2.69(\mathrm{dd}, J=16.3,8.5 \mathrm{~Hz}, 1 \mathrm{H}), 2.36(\mathrm{t}, J=8.1 \mathrm{~Hz}, 1 \mathrm{H})$, $1.98(\mathrm{t}, J=12.1 \mathrm{~Hz}, 1 \mathrm{H}), 0.88(\mathrm{~s}, 9 \mathrm{H}), 0.03(\mathrm{~s}, 6 \mathrm{H}) .{ }^{13} \mathrm{C}$ NMR $\left(100 \mathrm{MHz}, \mathrm{DMSO}-d_{6}\right) \delta$ $167.1,152.6,144.2,125.4,109.9,96.0,61.5,50.9,46.5,38.2,35.9,25.8,18.0,-5.36$, $-5.42$

methyl (4aS,7aS)-7-(((tert-butyldimethylsilyl)oxy)methyl)-1-oxo-1,4a,5,7a-tetrahydrocyclope nta[c]pyran-4-carboxylate, 2: ${ }^{1} \mathrm{H}$ NMR $\left(400 \mathrm{MHz}, \mathrm{DMSO}-d_{6}\right) \delta 7.60(\mathrm{~s}, 1 \mathrm{H}), 5.81(\mathrm{~s}$, $1 \mathrm{H}), 4.57-4.25(\mathrm{~m}, 2 \mathrm{H}), 3.83(\mathrm{~d}, J=10.4 \mathrm{~Hz}, 1 \mathrm{H}), 3.71(\mathrm{~s}, 3 \mathrm{H}), 3.38(\mathrm{q}, J=9.1 \mathrm{~Hz}, 1 \mathrm{H})$, $2.75(\mathrm{dd}, J=16.7,8.6 \mathrm{~Hz}, 1 \mathrm{H}), 2.14(\mathrm{dd}, J=16.1,7.9 \mathrm{~Hz}, 1 \mathrm{H}), 0.88(\mathrm{~s}, 9 \mathrm{H}), 0.05(\mathrm{~s}, 6 \mathrm{H})$. ${ }^{13} \mathrm{C}$ NMR $\left(100 \mathrm{MHz}, \mathrm{CDCl}_{3}\right) \delta 166.6,166.2,148.6,141.1,127.7,113.4,61.6,52.1,46.1$, $39.4,36.3,26.0,18.5,-5.3$.

methyl

(4aS,7aS)-7-(hydroxymethyl)-2-methyl-1-oxo-2,4a,5,7a-tetrahydro-1H-cyclopenta[c] pyridine-4-carboxylate, 3a. This compound was obtained as white solid in 49\% yield, $\mathrm{mp}=89-91{ }^{\circ} \mathrm{C} ;{ }^{1} \mathrm{H}$ NMR $\left(400 \mathrm{MHz}, \mathrm{CDCl}_{3}\right) \delta 7.18(\mathrm{~s}, 1 \mathrm{H}), 5.83-5.79(\mathrm{~m}, 1 \mathrm{H}), 4.32(\mathrm{q}$, $J=13.0 \mathrm{~Hz}, 2 \mathrm{H}), 4.16-4.04(\mathrm{~m}, 1 \mathrm{H}), 3.75(\mathrm{~s}, 3 \mathrm{H}), 3.70-3.64(\mathrm{~m}, 1 \mathrm{H}), 3.53(\mathrm{dt}, J=$ 11.0, 8.6 Hz, 1H), 3.17 (s, 3H), $2.97-2.82(\mathrm{~m}, 1 \mathrm{H}), 2.23$ (ddt, $J=16.6,8.8,2.0 \mathrm{~Hz}, 1 \mathrm{H})$. ${ }^{13} \mathrm{C} \mathrm{NMR}\left(100 \mathrm{MHz}, \mathrm{CDCl}_{3}\right) \delta 171.0,166.9,141.3,138.4,129.3,111.3,61.2,51.8,50.3$, 40.4, 37.6, 35.7. HRMS (ESI) calcd for $\mathrm{C}_{12} \mathrm{H}_{16} \mathrm{NO}_{4}[\mathrm{M}+\mathrm{H}]^{+}$238.1074, found 238.1075. 
methyl

(4aS,7aS)-7-(hydroxymethyl)-2-isopropyl-1-oxo-2,4a,5,7a-tetrahydro-1 $H$-cyclopenta

[c]pyridine-4-carboxylate, 3b. This compound was obtained as colorless oil in $61 \%$ yield; ${ }^{1} \mathrm{H}$ NMR $\left(400 \mathrm{MHz}, \mathrm{CDCl}_{3}\right) \delta 7.28(\mathrm{~s}, 1 \mathrm{H}), 5.84-5.80(\mathrm{~m}, 1 \mathrm{H}), 4.86$ (hept, $J=6.8$ $\mathrm{Hz}, 1 \mathrm{H}), 4.64(\mathrm{~s}, 1 \mathrm{H}), 4.32(\mathrm{q}, J=12.9 \mathrm{~Hz}, 2 \mathrm{H}), 3.76(\mathrm{~s}, 3 \mathrm{H}), 3.66(\mathrm{dt}, J=10.9,1.9 \mathrm{~Hz}$, 1H), $3.49(\mathrm{dt}, J=10.9,8.6 \mathrm{~Hz}, 1 \mathrm{H}), 2.93-2.82(\mathrm{~m}, 1 \mathrm{H}), 2.20(\mathrm{ddt}, J=16.6,8.7,2.0 \mathrm{~Hz}$, 1H), $1.21(\mathrm{dd}, J=9.7,6.9 \mathrm{~Hz}, 6 \mathrm{H}) .{ }^{13} \mathrm{C} \mathrm{NMR}\left(100 \mathrm{MHz}, \mathrm{CDCl}_{3}\right) \delta 170.1,167.0,141.3$, 132.7, 129.6, 111.8, 61.1, 51.8, 50.8, 45.4, 40.1, 37.0, 21.2, 20.7. HRMS (ESI) calcd for $\mathrm{C}_{14} \mathrm{H}_{20} \mathrm{NO}_{4}[\mathrm{M}+\mathrm{H}]^{+}$266.1387, found 266.1391.

methyl

(4aS,7aS)-2-butyl-7-(hydroxymethyl)-1-oxo-2,4a,5,7a-tetrahydro-1H-cyclopenta[c]p yridine-4-carboxylate, 3c. This compound was obtained as white solid in $72 \%$ yield, mp $=67-69{ }^{\circ} \mathrm{C} ;{ }^{1} \mathrm{H}$ NMR $\left(400 \mathrm{MHz}, \mathrm{CDCl}_{3}\right) \delta 7.20(\mathrm{~s}, 1 \mathrm{H}), 5.88-5.80(\mathrm{~m}, 1 \mathrm{H}), 5.00(\mathrm{~s}$, $1 \mathrm{H}), 4.42-4.23(\mathrm{~m}, 2 \mathrm{H}), 3.77(\mathrm{~s}, 3 \mathrm{H}), 3.71-3.64(\mathrm{~m}, 1 \mathrm{H}), 3.62-3.48(\mathrm{~m}, 3 \mathrm{H}), 2.93-$ $2.84(\mathrm{~m}, 1 \mathrm{H}), 2.23(\mathrm{ddt}, J=16.7,8.8,1.9 \mathrm{~Hz}, 1 \mathrm{H}), 1.62-1.52(\mathrm{~m}, 2 \mathrm{H}), 1.37-1.27(\mathrm{~m}$, 2H), $0.93(\mathrm{t}, J=7.3 \mathrm{~Hz}, 3 \mathrm{H}) .{ }^{13} \mathrm{C} \mathrm{NMR}\left(100 \mathrm{MHz}, \mathrm{CDCl}_{3}\right) \delta 170.6,166.8,140.7,137.4$, 129.8, 111.3, 60.9, 51.7, 50.5, 47.9, 40.0, 37.5, 30.7, 19.8, 13.7. HRMS (ESI) calcd for $\mathrm{C}_{15} \mathrm{H}_{22} \mathrm{NO}_{4}[\mathrm{M}+\mathrm{H}]^{+}$280.1543, found 280.1546.

methyl

(4aS,7aS)-2-cyclopropyl-7-(hydroxymethyl)-1-oxo-2,4a,5,7a-tetrahydro-1 $H$-cyclopen ta[c]pyridine-4-carboxylate, 3d. This compound was obtained as white solid in $61 \%$ yield, $\mathrm{mp}=82-84{ }^{\circ} \mathrm{C} ;{ }^{1} \mathrm{H} \mathrm{NMR}\left(400 \mathrm{MHz}, \mathrm{CDCl}_{3}\right) \delta 7.27(\mathrm{~s}, 1 \mathrm{H}), 5.85-5.75(\mathrm{~m}, 1 \mathrm{H})$, $4.31(\mathrm{q}, J=13.0 \mathrm{~Hz}, 3 \mathrm{H}), 3.75(\mathrm{~s}, 3 \mathrm{H}), 3.69-3.62(\mathrm{~m}, 1 \mathrm{H}), 3.49(\mathrm{dt}, J=11.1,8.6 \mathrm{~Hz}$, 
1H), $2.92-2.82(\mathrm{~m}, 2 \mathrm{H}), 2.19$ (ddt, $J=16.7,8.6,2.0 \mathrm{~Hz}, 1 \mathrm{H}), 0.99-0.91(\mathrm{~m}, 2 \mathrm{H}), 0.74$ $-0.65(\mathrm{~m}, 2 \mathrm{H}) .{ }^{13} \mathrm{C} \mathrm{NMR}\left(100 \mathrm{MHz}, \mathrm{CDCl}_{3}\right) \delta 172.2,166.9,141.1,137.7,129.5,111.1$, $61.1,51.8,50.9,40.2,37.2,30.7,7.3,7.2$. HRMS (ESI) calcd for $\mathrm{C}_{14} \mathrm{H}_{18} \mathrm{NO}_{4}[\mathrm{M}+\mathrm{H}]^{+}$ 264.1230, found 264.1234.

methyl

(4aS,7aS)-2-(3-chloropropyl)-7-(hydroxymethyl)-1-oxo-2,4a,5,7a-tetrahydro-1 $H$-cycl openta[c]pyridine-4-carboxylate, 3 e. This compound was obtained as yellow oil in $62 \%$ yield; ${ }^{1} \mathrm{H}$ NMR (400 MHz, $\left.\mathrm{CDCl}_{3}\right) \delta 7.20(\mathrm{~s}, 1 \mathrm{H}), 5.93-5.73(\mathrm{~m}, 2 \mathrm{H}), 4.39-4.23(\mathrm{~m}$, $2 \mathrm{H}), 3.78-3.60(\mathrm{~m}, 6 \mathrm{H}), 3.50(\mathrm{dtt}, J=9.4,6.9,3.4 \mathrm{~Hz}, 3 \mathrm{H}), 2.94-2.81(\mathrm{~m}, 1 \mathrm{H}), 2.24-$ $2.13(\mathrm{~m}, 1 \mathrm{H}), 2.10-2.00(\mathrm{~m}, 2 \mathrm{H}) .{ }^{13} \mathrm{C} \mathrm{NMR}\left(100 \mathrm{MHz}, \mathrm{CDCl}_{3}\right) \delta 170.8,166.7,140.7$, 137.4, 129.7, 111.6, 60.9, 51.8, 50.3, 46.0, 41.8, 40.1, 37.5, 31.1. HRMS (ESI) calcd for $\mathrm{C}_{14} \mathrm{H}_{19} \mathrm{ClNO}_{4}[\mathrm{M}+\mathrm{H}]^{+}$300.0997, found 300.1000 .

methyl (4aS,7aS)-7-(hydroxymethyl)-2-(2-(methylsulfonyl)ethyl)-1-oxo-2,4a,5,7a-tetrahydro -1H-cyclopenta[c]pyridine-4-carboxylate, 3f. This compound was obtained as white solid in $66 \%$ yield, $\mathrm{mp}=134-135{ }^{\circ} \mathrm{C} ;{ }^{1} \mathrm{H}$ NMR $\left(400 \mathrm{MHz}, \mathrm{CDCl}_{3}\right) \delta 7.26(\mathrm{~s}, 1 \mathrm{H}), 5.85$ (d, $J=2.8 \mathrm{~Hz}, 1 \mathrm{H}), 4.33(\mathrm{q}, J=13.2 \mathrm{~Hz}, 2 \mathrm{H}), 4.04(\mathrm{dtt}, J=21.0,14.2,6.8 \mathrm{~Hz}, 2 \mathrm{H}), 3.76$ (s, 3H), $3.70(\mathrm{~d}, J=10.8 \mathrm{~Hz}, 1 \mathrm{H}), 3.56(\mathrm{dt}, J=11.0,8.6 \mathrm{~Hz}, 1 \mathrm{H}), 3.36(\mathrm{td}, J=6.7,2.7 \mathrm{~Hz}$, 2H), $3.03-2.82(\mathrm{~m}, 5 \mathrm{H}), 2.27(\mathrm{ddt}, J=16.7,8.5,1.9 \mathrm{~Hz}, 1 \mathrm{H}) .{ }^{13} \mathrm{C} \mathrm{NMR}(100 \mathrm{MHz}$, $\left.\mathrm{CDCl}_{3}\right) \delta 171.2,166.4,140.5,137.0,130.0,112.3,60.9,52.1,51.8,50.2,42.6,41.7,39.9$, 37.3. HRMS (ESI) calcd for $\mathrm{C}_{14} \mathrm{H}_{20} \mathrm{NO}_{6} \mathrm{~S}[\mathrm{M}+\mathrm{H}]^{+} 330.1006$, found 330.1004 .

2-((4aS,7aS)-7-(hydroxymethyl)-4-(methoxycarbonyl)-1-oxo-1,4a,5,7a-tetrahydro-2 $\boldsymbol{H}$-cyclopenta[c]pyridin-2-yl)ethane-1-sulfonic acid, 3g. This compound was obtained 
as colorless oil in $70 \%$ yield; ${ }^{1} \mathrm{H}$ NMR (400 MHz, MeOD) $\delta 7.43(\mathrm{~s}, 1 \mathrm{H}), 5.82(\mathrm{~s}, 1 \mathrm{H})$, 4.37 (t, $J=10.0 \mathrm{~Hz}, 2 \mathrm{H}), 3.98$ (h, $J=7.2 \mathrm{~Hz}, 2 \mathrm{H}), 3.75(\mathrm{~s}, 3 \mathrm{H}), 3.70(\mathrm{~d}, J=11.1 \mathrm{~Hz}, 1 \mathrm{H})$, $3.55-3.45(\mathrm{~m}, 1 \mathrm{H}), 3.35(\mathrm{~s}, 1 \mathrm{H}), 3.09(\mathrm{t}, J=6.9 \mathrm{~Hz}, 2 \mathrm{H}), 2.83(\mathrm{dd}, J=16.5,8.5 \mathrm{~Hz}, 1 \mathrm{H})$, $2.28-2.12(\mathrm{~m}, 1 \mathrm{H}) .{ }^{13} \mathrm{C}$ NMR (100 MHz, MeOD) $\delta 171.6,168.9,143.5,139.7,128.5$, $111.9,61.5,52.2,50.0,49.9,45.2,40.8,38.4$. HRMS (ESI) calcd for $\mathrm{C}_{13} \mathrm{H}_{18} \mathrm{NO}_{7} \mathrm{~S}$ $[\mathrm{M}+\mathrm{H}]^{+}$332.0798, found 332.0802.

methyl

(4aS,7aS)-2-allyl-7-(hydroxymethyl)-1-oxo-2,4a,5,7a-tetrahydro-1 $H$-cyclopenta[c]py ridine-4-carboxylate, $\mathbf{3 h}$. This compound was obtained as white solid in $80 \%$ yield, $\mathrm{mp}$ $=60-62{ }^{\circ} \mathrm{C} ;{ }^{1} \mathrm{H}$ NMR $\left(400 \mathrm{MHz}, \mathrm{CDCl}_{3}\right) \delta 7.16(\mathrm{~s}, 1 \mathrm{H}), 5.87-5.73(\mathrm{~m}, 2 \mathrm{H}), 5.26-5.14$ (m, 2H), $4.62(\mathrm{~s}, 1 \mathrm{H}), 4.33$ (q, $J=13.1 \mathrm{~Hz}, 2 \mathrm{H}), 4.18(\mathrm{ddt}, J=5.4,3.5,1.6 \mathrm{~Hz}, 2 \mathrm{H}), 3.75$ (s, 3H), $3.70(\mathrm{dt}, J=10.8,2.0 \mathrm{~Hz}, 1 \mathrm{H}), 3.54(\mathrm{dt}, J=10.9,8.7 \mathrm{~Hz}, 1 \mathrm{H}), 2.96-2.84(\mathrm{~m}$, 1H), 2.24 (ddt, $J=16.6,8.8,2.0 \mathrm{~Hz}, 1 \mathrm{H}) .{ }^{13} \mathrm{C} \mathrm{NMR}\left(100 \mathrm{MHz}, \mathrm{CDCl}_{3}\right) \delta 170.3,166.7$, 141.0, 136.8, 131.7, 129.5, 118.4, 111.5, 61.0, 51.7, 50.3, 49.6, 40.1, 37.5. HRMS (ESI) calcd for $\mathrm{C}_{14} \mathrm{H}_{18} \mathrm{NO}_{4}[\mathrm{M}+\mathrm{H}]^{+}$264.1230, found 264.1229.

methyl

(4aS,7aS)-7-(hydroxymethyl)-1-oxo-2-(prop-2-yn-1-yl)-2,4a,5,7a-tetrahydro-1H-cycl openta[c]pyridine-4-carboxylate, 3i. This compound was obtained as white solid in $70 \%$ yield, $\mathrm{mp}=83-84{ }^{\circ} \mathrm{C} ;{ }^{1} \mathrm{H}$ NMR $\left(400 \mathrm{MHz}, \mathrm{CDCl}_{3}\right) \delta 7.41(\mathrm{~s}, 1 \mathrm{H}), 5.91-5.78(\mathrm{~m}$, 1H), $4.48(\mathrm{dd}, J=17.5,2.6 \mathrm{~Hz}, 1 \mathrm{H}), 4.42-4.27(\mathrm{~m}, 3 \mathrm{H}), 3.78(\mathrm{~s}, 3 \mathrm{H}), 3.75-3.69(\mathrm{~m}$, 1H), $3.57(\mathrm{dt}, J=11.0,8.7 \mathrm{~Hz}, 2 \mathrm{H}), 2.99-2.85(\mathrm{~m}, 1 \mathrm{H}), 2.36(\mathrm{t}, J=2.6 \mathrm{~Hz}, 1 \mathrm{H}), 2.26$ (ddt, $J=16.6,8.8,2.0 \mathrm{~Hz}, 1 \mathrm{H}) .{ }^{13} \mathrm{C}$ NMR $\left(100 \mathrm{MHz}, \mathrm{CDCl}_{3}\right) \delta 170.2,166.7,140.9,135.7$, $129.8,112.5,77.1,74.0,61.1,51.9,50.4,40.3,37.5,36.4$. HRMS (ESI) calcd for 
$\mathrm{C}_{14} \mathrm{H}_{16} \mathrm{NO}_{4}[\mathrm{M}+\mathrm{H}]^{+}$262.1074, found 262.1075.

methyl

(4aS,7aS)-7-(hydroxymethyl)-2-((S)-1-methoxy-1-oxopropan-2-yl)-1-oxo-2,4a,5,7a-te trahydro- $\mathbf{H}$-cyclopenta $[\boldsymbol{c}]$ pyridine-4-carboxylate, $\mathbf{3} \mathbf{j}$. This compound was obtained as colorless oil in 55\% yield; ${ }^{1} \mathrm{H}$ NMR (400 MHz, $\left.\mathrm{CDCl}_{3}\right) \delta 7.19(\mathrm{~s}, 1 \mathrm{H}), 5.78(\mathrm{~s}, 1 \mathrm{H}), 5.11$ (q, $J=7.3 \mathrm{~Hz}, 1 \mathrm{H}), 4.35-4.18(\mathrm{~m}, 2 \mathrm{H}), 3.85-3.64(\mathrm{~m}, 8 \mathrm{H}), 3.50(\mathrm{q}, J=10.4,9.6 \mathrm{~Hz}$, 1H), $2.84(\mathrm{dd}, J=16.7,8.6 \mathrm{~Hz}, 1 \mathrm{H}), 2.17(\mathrm{dd}, J=16.5,8.5 \mathrm{~Hz}, 1 \mathrm{H}), 1.46(\mathrm{~d}, J=7.4 \mathrm{~Hz}$, 3H). ${ }^{13} \mathrm{C} \mathrm{NMR}\left(100 \mathrm{MHz}, \mathrm{CDCl}_{3}\right) \delta 170.9,170.1,166.5,141.1,134.1,128.8,111.7,60.7$, 52.6, 52.2, 51.5, 49.8, 39.8, 36.7, 15.7. HRMS (ESI) calcd for $\mathrm{C}_{15} \mathrm{H}_{19} \mathrm{NNaO}_{6}[\mathrm{M}+\mathrm{Na}]^{+}$ 332.1105 , found 332.1111 .

methyl

(4aS,7aS)-2-((S)-1-ethoxy-1-oxopropan-2-yl)-7-(hydroxymethyl)-1-oxo-2,4a,5,7a-tetr ahydro-1H-cyclopenta[c]pyridine-4-carboxylate, $3 \mathrm{k}$. This compound was obtained as colorless oil in $61 \%$ yield; ${ }^{1} \mathrm{H}$ NMR $\left(400 \mathrm{MHz}, \mathrm{CDCl}_{3}\right) \delta 7.20(\mathrm{~s}, 1 \mathrm{H}), 5.85-5.66(\mathrm{~m}$, 2H), $5.04(\mathrm{q}, J=7.4 \mathrm{~Hz}, 1 \mathrm{H}), 4.36-4.20(\mathrm{~m}, 2 \mathrm{H}), 4.13(\mathrm{qd}, J=7.1,5.2 \mathrm{~Hz}, 2 \mathrm{H}), 3.74-$ 3.64 (m, 4H), 3.49 (dt, $J=10.9,8.7 \mathrm{~Hz}, 1 \mathrm{H}), 2.91-2.76$ (m, 1H), 2.17 (ddt, $J=16.6,8.8$, $2.0 \mathrm{~Hz}, 1 \mathrm{H}), 1.46(\mathrm{~d}, J=7.4 \mathrm{~Hz}, 3 \mathrm{H}), 1.19(\mathrm{t}, J=7.1 \mathrm{~Hz}, 3 \mathrm{H}) .{ }^{13} \mathrm{C} \mathrm{NMR}(100 \mathrm{MHz}$, $\left.\mathrm{CDCl}_{3}\right) \delta 170.5,170.4,166.6,140.7,134.4,129.7,111.9,61.8,60.8,52.7,51.7,50.2$, 39.9, 36.9, 15.8, 14.0. HRMS (ESI) calcd for $\mathrm{C}_{16} \mathrm{H}_{22} \mathrm{NO}_{6}[\mathrm{M}+\mathrm{H}]^{+}$324.1442, found 324.1445 .

methyl

(4aS,7aS)-2-((S)-3-(1H-indol-3-yl)-1-methoxy-1-oxopropan-2-yl)-7-(hydroxymethyl)1-oxo-2,4a,5,7a-tetrahydro-1 $H$-cyclopenta[c]pyridine-4-carboxylate, 31. This 
compound was obtained as yellow oil in $46 \%$ yield; ${ }^{1} \mathrm{H}$ NMR $\left(400 \mathrm{MHz}, \mathrm{CDCl}_{3}\right) \delta 8.56$ (s, 1H), $7.56(\mathrm{~d}, J=7.7 \mathrm{~Hz}, 1 \mathrm{H}), 7.37-7.30(\mathrm{~m}, 1 \mathrm{H}), 7.20(\mathrm{ddd}, J=8.2,7.0,1.2 \mathrm{~Hz}, 1 \mathrm{H})$, $7.12(\mathrm{td}, J=7.4,6.9,1.0 \mathrm{~Hz}, 1 \mathrm{H}), 6.97(\mathrm{~d}, J=2.3 \mathrm{~Hz}, 1 \mathrm{H}), 5.65(\mathrm{~s}, 1 \mathrm{H}), 5.59$ (dd, $J=$ 10.9, $5.3 \mathrm{~Hz}, 1 \mathrm{H}), 4.30-4.14(\mathrm{~m}, 1 \mathrm{H}), 4.14-4.01(\mathrm{~m}, 2 \mathrm{H}), 3.85(\mathrm{~s}, 3 \mathrm{H}), 3.76(\mathrm{~s}, 3 \mathrm{H})$, $3.70-3.56(\mathrm{~m}, 2 \mathrm{H}), 3.43(\mathrm{dt}, J=10.7,8.4 \mathrm{~Hz}, 1 \mathrm{H}), 3.34(\mathrm{dd}, J=15.3,10.9 \mathrm{~Hz}, 1 \mathrm{H})$, $2.75-2.61(\mathrm{~m}, 1 \mathrm{H}), 1.70(\mathrm{dd}, J=16.6,8.1 \mathrm{~Hz}, 1 \mathrm{H}), 1.00-0.82(\mathrm{~m}, 1 \mathrm{H}) .{ }^{13} \mathrm{C}$ NMR $(100$ $\left.\mathrm{MHz}, \mathrm{CDCl}_{3}\right) \delta 170.5,170.4,166.7,140.4,136.2,134.7,129.2,127.0,122.9,122.2$, 119.6, 118.4, 111.7, 111.3, 109.6, 60.6, 56.9, 52.9, 51.7, 50.3, 39.5, 36.6, 26.0. HRMS (ESI) calcd for $\mathrm{C}_{23} \mathrm{H}_{25} \mathrm{~N}_{2} \mathrm{O}_{6}[\mathrm{M}+\mathrm{H}]^{+} 425.1707$, found 425.1710 .

\section{methyl}

(4aS,7aS)-7-(hydroxymethyl)-1-oxo-2-((tetrahydrofuran-2-yl)methyl)-2,4a,5,7a-tetra hydro-1H-cyclopenta[c]pyridine-4-carboxylate, $\mathbf{3 m}$. This compound was obtained as white solid in $60 \%$ yield, $\mathrm{mp}=72-74{ }^{\circ} \mathrm{C} ;{ }^{1} \mathrm{H}$ NMR $\left(400 \mathrm{MHz}, \mathrm{CDCl}_{3}\right) \delta 7.32(\mathrm{~d}, J=4.3$ Hz, 1H), $5.81(\mathrm{~s}, 1 \mathrm{H}), 4.36-4.22(\mathrm{~m}, 2 \mathrm{H}), 4.18-3.98(\mathrm{~m}, 2 \mathrm{H}), 3.93-3.80(\mathrm{~m}, 2 \mathrm{H}), 3.77$ $-3.63(\mathrm{~m}, 5 \mathrm{H}), 3.51(\mathrm{dtd}, J=10.6,8.6,1.8 \mathrm{~Hz}, 1 \mathrm{H}), 3.39(\mathrm{td}, J=13.5,7.6 \mathrm{~Hz}, 1 \mathrm{H}), 2.88$ (ddd, $J=16.7,7.5,3.5 \mathrm{~Hz}, 1 \mathrm{H}$ ), 2.23 (dddt, $J=18.5,16.5,8.6,1.9 \mathrm{~Hz}, 1 \mathrm{H}$ ), 1.97 (dtd, $J$ $=12.2,6.9,6.4,2.4 \mathrm{~Hz}, 1 \mathrm{H}), 1.92-1.80(\mathrm{~m}, 2 \mathrm{H}), 1.48(\mathrm{dp}, J=12.0,7.9 \mathrm{~Hz}, 1 \mathrm{H}) .{ }^{13} \mathrm{C}$ NMR $\left(100 \mathrm{MHz}, \mathrm{CDCl}_{3}\right) \delta 170.9,167.0,141.3,138.6,129.6,110.8,77.3,68.3,61.1$, $51.7,51.2,50.3,40.1,37.3,29.0,25.7$. HRMS (ESI) calcd for $\mathrm{C}_{16} \mathrm{H}_{22} \mathrm{NO}_{5}[\mathrm{M}+\mathrm{H}]^{+}$ 308.1492, found 308.1494.

methyl (4aS,7aS)-7-(hydroxymethyl)-1-oxo-2-(piperidin-1-yl)-2,4a,5,7a-tetrahydro-1H-cyclo penta[c]pyridine-4-carboxylate, $3 \mathbf{n}$. This compound was obtained as white solid in $40 \%$ 
yield, $\mathrm{mp}=78-80{ }^{\circ} \mathrm{C} ;{ }^{1} \mathrm{H}$ NMR $\left(400 \mathrm{MHz}, \mathrm{CDCl}_{3}\right) \delta 7.35(\mathrm{~s}, 1 \mathrm{H}), 5.87-5.80(\mathrm{~m}, 1 \mathrm{H})$, $4.47-4.18(\mathrm{~m}, 2 \mathrm{H}), 3.76(\mathrm{~s}, 3 \mathrm{H}), 3.72(\mathrm{~d}, J=11.1 \mathrm{~Hz}, 1 \mathrm{H}), 3.62-3.30(\mathrm{~m}, 4 \mathrm{H}), 3.06-$ $2.77(\mathrm{~m}, 3 \mathrm{H}), 2.22(\mathrm{ddt}, J=16.6,8.7,2.0 \mathrm{~Hz}, 1 \mathrm{H}), 1.88-1.47(\mathrm{~m}, 6 \mathrm{H}) .{ }^{13} \mathrm{C}$ NMR $(100$ $\left.\mathrm{MHz}, \mathrm{CDCl}_{3}\right) \delta 170.2,166.8,141.0,140.0,129.8,110.9,61.1,52.2,51.8,40.1,37.2$, 26.6, 23.3. HRMS (ESI) calcd for $\mathrm{C}_{16} \mathrm{H}_{23} \mathrm{~N}_{2} \mathrm{O}_{4}[\mathrm{M}+\mathrm{H}]^{+}$307.1652, found 307.1653.

methyl

(4aS,7aS)-2-cyclohexyl-7-(hydroxymethyl)-1-oxo-2,4a,5,7a-tetrahydro-1 $H$-cyclopent

a $[c]$ pyridine-4-carboxylate, 3o. This compound was obtained as yellow oil in 93\% yield; ${ }^{1} \mathrm{H}$ NMR (400 MHz, $\left.\mathrm{CDCl}_{3}\right) \delta 7.30(\mathrm{~s}, 1 \mathrm{H}), 7.28-7.18(\mathrm{~m}, 1 \mathrm{H}), 5.85(\mathrm{dd}, J=3.0,1.5 \mathrm{~Hz}$, 1H), $4.53-4.21(\mathrm{~m}, 3 \mathrm{H}), 3.76(\mathrm{~s}, 3 \mathrm{H}), 3.66(\mathrm{dt}, J=11.0,1.8 \mathrm{~Hz}, 1 \mathrm{H}), 3.48(\mathrm{dt}, J=10.9$, $8.7 \mathrm{~Hz}, 1 \mathrm{H}), 2.86$ (ddt, $J=17.1,8.8,2.2 \mathrm{~Hz}, 1 \mathrm{H}), 2.20$ (ddt, $J=16.6,9.0,1.9 \mathrm{~Hz}, 1 \mathrm{H})$, $1.93-1.77(\mathrm{~m}, 2 \mathrm{H}), 1.77-1.63(\mathrm{~m}, 3 \mathrm{H}), 1.46-1.31(\mathrm{~m}, 4 \mathrm{H}), 1.22-1.07(\mathrm{~m}, 1 \mathrm{H}) .{ }^{13} \mathrm{C}$ NMR $\left(100 \mathrm{MHz}, \mathrm{CDCl}_{3}\right) \delta 170.5,167.0,140.4,133.4,130.5,111.5,60.8,53.5,51.8$, 51.0, 40.0, 37.0, 31.7, 31.0, 25.8, 25.7, 25.3. HRMS (ESI) calcd for $\mathrm{C}_{17} \mathrm{H}_{24} \mathrm{NO}_{4}[\mathrm{M}+\mathrm{H}]^{+}$ 306.1700, found 306.1703.

methyl

(4aS,7aS)-7-(hydroxymethyl)-1-oxo-2-(tetrahydro-2H-pyran-4-yl)-2,4a,5,7a-tetrahyd ro-1H-cyclopenta[c]pyridine-4-carboxylate, 3p. This compound was obtained as colorless oil in $45 \%$ yield; ${ }^{1} \mathrm{H}$ NMR $\left(400 \mathrm{MHz}, \mathrm{CDCl}_{3}\right) \delta 7.33(\mathrm{~s}, 1 \mathrm{H}), 5.81(\mathrm{~s}, 1 \mathrm{H}), 4.61$ (s, 1H), $4.30(\mathrm{q}, J=13.1 \mathrm{~Hz}, 2 \mathrm{H}), 3.74(\mathrm{~s}, 3 \mathrm{H}), 3.69(\mathrm{dt}, J=10.8,2.0 \mathrm{~Hz}, 1 \mathrm{H}), 3.50-$ $3.25(\mathrm{~m}, 3 \mathrm{H}), 3.06-2.77(\mathrm{~m}, 3 \mathrm{H}), 2.20(\mathrm{ddt}, J=16.6,8.7,1.8 \mathrm{~Hz}, 1 \mathrm{H}), 1.76-1.41(\mathrm{~m}$, 5H). ${ }^{13} \mathrm{C}$ NMR (100 MHz, $\left.\mathrm{CDCl}_{3}\right) \delta 170.1,166.7,140.9,139.9,129.7,110.8,61.0,52.0$, 51.7, 40.0, 37.1, 26.5, 23.2. HRMS (ESI) calcd for $\mathrm{C}_{16} \mathrm{H}_{22} \mathrm{NO}_{5}[\mathrm{M}+\mathrm{H}]^{+}$308.1492, found 
308.1493.

methyl

(4aS,7aS)-2-(4-hydroxycyclohexyl)-7-(hydroxymethyl)-1-oxo-2,4a,5,7a-tetrahydro-1

$\boldsymbol{H}$-cyclopenta[c]pyridine-4-carboxylate, 3q. This compound was obtained as colorless oil in $69 \%$ yield; ${ }^{1} \mathrm{H}$ NMR (400 MHz, $\left.\mathrm{CDCl}_{3}\right) \delta 7.23(\mathrm{~s}, 1 \mathrm{H}), 5.81(\mathrm{~s}, 1 \mathrm{H}), 4.44(\mathrm{tt}, J=$ 12.1, $3.9 \mathrm{~Hz}, 1 \mathrm{H}), 4.30$ (q, $J=13.0 \mathrm{~Hz}, 2 \mathrm{H}), 4.21-4.09$ (m, 2H), $3.74(\mathrm{~s}, 3 \mathrm{H}), 3.68-$ $3.56(\mathrm{~m}, 2 \mathrm{H}), 3.47$ (dt, $J=10.9,8.6 \mathrm{~Hz}, 1 \mathrm{H}), 2.92-2.80(\mathrm{~m}, 1 \mathrm{H}), 2.17$ (ddt, $J=16.6,8.7$, $1.9 \mathrm{~Hz}, 1 \mathrm{H}), 2.11-1.98(\mathrm{~m}, 2 \mathrm{H}), 1.79-1.66(\mathrm{~m}, 2 \mathrm{H}), 1.64-1.35(\mathrm{~m}, 4 \mathrm{H}) .{ }^{13} \mathrm{C}$ NMR $\left(100 \mathrm{MHz}, \mathrm{CDCl}_{3}\right) \delta 170.4,167.0,140.9,133.1,129.8,111.6,69.5,60.9,52.4,51.8,50.7$, 40.0, 36.8, 34.33, 34.25, 29.2, 28.6. HRMS (ESI) calcd for $\mathrm{C}_{17} \mathrm{H}_{24} \mathrm{NO}_{5}[\mathrm{M}+\mathrm{H}]^{+}$322.1649, found 322.1656 .

methyl

(4aS,7aS)-7-(hydroxymethyl)-1-oxo-2-(4-(trifluoromethyl)cyclohexyl)-2,4a,5,7a-tetra hydro-1H-cyclopenta[c]pyridine-4-carboxylate, $3 r$. This compound was obtained as colorless oil in $68 \%$ yield; ${ }^{1} \mathrm{H}$ NMR $\left(400 \mathrm{MHz}, \mathrm{CDCl}_{3}\right) \delta 7.23(\mathrm{~s}, 1 \mathrm{H}), 5.80(\mathrm{~s}, 1 \mathrm{H}), 4.50$ (dt, $J=11.5,6.3 \mathrm{~Hz}, 1 \mathrm{H}), 4.39-4.23(\mathrm{~m}, 3 \mathrm{H}), 3.73(\mathrm{~s}, 3 \mathrm{H}), 3.65$ (dt, $J=10.9,1.8 \mathrm{~Hz}$, 1H), $3.47(\mathrm{dt}, J=10.9,8.6 \mathrm{~Hz}, 1 \mathrm{H}), 2.92-2.78(\mathrm{~m}, 1 \mathrm{H}), 2.40-2.24(\mathrm{~m}, 1 \mathrm{H}), 2.23-2.06$ (m, 3H), $1.78-1.57(\mathrm{~m}, 6 \mathrm{H}) .{ }^{13} \mathrm{C}$ NMR $\left(100 \mathrm{MHz}, \mathrm{CDCl}_{3}\right) \delta 170.2,166.8,141.4,132.8$, $129.3,128.53(\mathrm{q}, J=281.1 \mathrm{~Hz}), 111.7,61.1,51.8,51.7,50.6,40.1,36.8,35.4(\mathrm{q}, J=25.9$ $\mathrm{Hz}), 26.5,25.9,23.43,23.39$. HRMS (ESI) calcd for $\mathrm{C}_{18} \mathrm{H}_{23} \mathrm{~F}_{3} \mathrm{NO}_{4}[\mathrm{M}+\mathrm{H}]^{+} 374.1574$, found 374.1574 .

methyl

(4aS,7aS)-2-(4-(tert-butyl)cyclohexyl)-7-(hydroxymethyl)-1-oxo-2,4a,5,7a-tetrahydro 
-1H-cyclopenta[c]pyridine-4-carboxylate, 3s. This compound was obtained as colorless oil in 47\% yield; ${ }^{1} \mathrm{H}$ NMR $\left(400 \mathrm{MHz}, \mathrm{CDCl}_{3}\right) \delta 7.27(\mathrm{~s}, 1 \mathrm{H}), 5.80(\mathrm{~s}, 1 \mathrm{H}), 5.36(\mathrm{~s}, 1 \mathrm{H})$, $4.40(\mathrm{dt}, J=12.3,3.8 \mathrm{~Hz}, 1 \mathrm{H}), 4.30(\mathrm{q}, J=13.3 \mathrm{~Hz}, 2 \mathrm{H}), 3.73(\mathrm{~s}, 3 \mathrm{H}), 3.64(\mathrm{dq}, J=11.6$, 2.8, $2.3 \mathrm{~Hz}, 1 \mathrm{H}), 3.46(\mathrm{dt}, J=11.0,8.7 \mathrm{~Hz}, 1 \mathrm{H}), 2.90-2.79(\mathrm{~m}, 1 \mathrm{H}), 2.18(\mathrm{ddq}, J=16.5$, 8.8, $1.9 \mathrm{~Hz}, 1 \mathrm{H}), 1.91-1.79(\mathrm{~m}, 2 \mathrm{H}), 1.79-1.59(\mathrm{~m}, 2 \mathrm{H}), 1.53-1.07(\mathrm{~m}, 4 \mathrm{H}), 1.02(\mathrm{dt}$, $J=12.0,2.9 \mathrm{~Hz}, 1 \mathrm{H}), 0.83(\mathrm{~s}, 9 \mathrm{H}) .{ }^{13} \mathrm{C} \mathrm{NMR}\left(100 \mathrm{MHz}, \mathrm{CDCl}_{3}\right) \delta 170.3,167.0,141.0$ $133.3,129.7,111.3,61.0,53.4,51.7,50.8,47.0,40.0,36.9,32.4,31.6,31.0,27.6,26.5$, 26.4. HRMS (ESI) calcd for $\mathrm{C}_{21} \mathrm{H}_{32} \mathrm{NO}_{4}[\mathrm{M}+\mathrm{H}]^{+}$362.2326, found 362.2331.

methyl

(4aS,7aS)-7-(hydroxymethyl)-2-(4-methylcyclohexyl)-1-oxo-2,4a,5,7a-tetrahydro-1H -cyclopenta[c]pyridine-4-carboxylate, 3t. This compound was obtained as colorless oil in $92 \%$ yield; ${ }^{1} \mathrm{H}$ NMR $\left(400 \mathrm{MHz}, \mathrm{CDCl}_{3}\right) \delta 7.26(\mathrm{~s}, 1 \mathrm{H}), 5.77(\mathrm{~s}, 1 \mathrm{H}), 4.44-4.34(\mathrm{~m}$, $1 \mathrm{H}), 4.28(\mathrm{q}, J=13.1 \mathrm{~Hz}, 2 \mathrm{H}), 3.72(\mathrm{~s}, 3 \mathrm{H}), 3.63(\mathrm{~d}, J=10.8 \mathrm{~Hz}, 1 \mathrm{H}), 3.45(\mathrm{dtd}, J=10.5$, 8.5, 1.7 Hz, 1H), $2.83(\mathrm{dd}, J=16.8,8.5 \mathrm{~Hz}, 1 \mathrm{H}), 2.21-2.10(\mathrm{~m}, 1 \mathrm{H}), 1.83-1.70(\mathrm{~m}$, $2 \mathrm{H}), 1.70-1.61(\mathrm{~m}, 2 \mathrm{H}), 1.55-1.39(\mathrm{~m}, 2 \mathrm{H}), 1.39-1.29(\mathrm{~m}, 1 \mathrm{H}), 1.25-1.17(\mathrm{~m}, 1 \mathrm{H})$, $1.14-1.00(\mathrm{~m}, 2 \mathrm{H}), 0.87(\mathrm{~d}, J=6.4 \mathrm{~Hz}, 3 \mathrm{H}) .{ }^{13} \mathrm{C} \mathrm{NMR}\left(100 \mathrm{MHz}, \mathrm{CDCl}_{3}\right) \delta 170.2$ $167.0,141.4,133.4,129.2,111.2,61.0,53.1,51.7,50.7,40.0,36.8,34.12,34.05,31.8$, 31.2, 30.6, 22.1. HRMS (ESI) calcd for $\mathrm{C}_{18} \mathrm{H}_{26} \mathrm{NO}_{4}[\mathrm{M}+\mathrm{H}]^{+} 320.1856$, found 320.1860 .

methyl

(4aS,7aS)-2-(1-(tert-butoxycarbonyl)piperidin-4-yl)-7-(hydroxymethyl)-1-oxo-2,4a,5, 7a-tetrahydro-1H-cyclopenta[c]pyridine-4-carboxylate, $3 \mathbf{3 u}$. This compound was obtained as colorless oil in 77\% yield; ${ }^{1} \mathrm{H}$ NMR $\left(400 \mathrm{MHz}, \mathrm{CDCl}_{3}\right) \delta 7.24(\mathrm{~s}, 1 \mathrm{H}), 5.83$ (s, 1H), $4.68-4.58(\mathrm{~m}, 1 \mathrm{H}), 4.39-4.15(\mathrm{~m}, 4 \mathrm{H}), 3.76(\mathrm{~s}, 3 \mathrm{H}), 3.70(\mathrm{~d}, J=11.0 \mathrm{~Hz}, 1 \mathrm{H})$, 
$3.51(\mathrm{dt}, J=10.9,8.6 \mathrm{~Hz}, 1 \mathrm{H}), 3.34(\mathrm{~s}, 1 \mathrm{H}), 2.94-2.85(\mathrm{~m}, 1 \mathrm{H}), 2.84-2.70(\mathrm{~m}, 2 \mathrm{H})$, $2.21(\mathrm{dd}, J=16.7,8.7 \mathrm{~Hz}, 1 \mathrm{H}), 1.75-1.53(\mathrm{~m}, 4 \mathrm{H}), 1.47(\mathrm{~s}, 9 \mathrm{H}) .{ }^{13} \mathrm{C}$ NMR $(100 \mathrm{MHz}$, $\left.\mathrm{CDCl}_{3}\right) \delta 170.3,166.8,154.6,141.2,132.7,129.7,112.0,80.2,61.1,51.9,51.5,50.8$, 40.2, 36.8, 30.6, 30.0, 28.5. HRMS (ESI) calcd for $\mathrm{C}_{21} \mathrm{H}_{31} \mathrm{~N}_{2} \mathrm{O}_{6}[\mathrm{M}+\mathrm{H}]^{+}$407.2177, found 407.2166.

methyl

(4aS,7aS)-2-benzyl-7-(hydroxymethyl)-1-oxo-2,4a,5,7a-tetrahydro-1H-cyclopenta[c] pyridine-4-carboxylate, 3v. This compound was obtained as white solid in 59\% yield, $\mathrm{mp}=71-73{ }^{\circ} \mathrm{C} ;{ }^{1} \mathrm{H}$ NMR $\left(400 \mathrm{MHz}, \mathrm{CDCl}_{3}\right) \delta 7.41-7.31(\mathrm{~m}, 3 \mathrm{H}), 7.29(\mathrm{~s}, 1 \mathrm{H}), 7.28-$ $7.23(\mathrm{~m}, 2 \mathrm{H}), 5.92-5.83(\mathrm{~m}, 1 \mathrm{H}), 4.92-4.67(\mathrm{~m}, 2 \mathrm{H}), 4.47-4.28(\mathrm{~m}, 2 \mathrm{H}), 3.81-3.73$ (m, 4H), 3.59 (dt, $J=10.9,8.6 \mathrm{~Hz}, 1 \mathrm{H}), 3.00-2.87$ (m, 1H), 2.28 (ddt, $J=16.6,8.8,1.9$ $\mathrm{Hz}, 1 \mathrm{H}) .{ }^{13} \mathrm{C} \mathrm{NMR}\left(100 \mathrm{MHz}, \mathrm{CDCl}_{3}\right) \delta 170.8,166.8,141.3,137.1,136.1,129.5,129.1$, 128.2, 127.7, 111.9, 61.2, 51.8, 50.8, 50.5, 40.2, 37.6. HRMS (ESI) calcd for $\mathrm{C}_{18} \mathrm{H}_{20} \mathrm{NO}_{4}$ $[\mathrm{M}+\mathrm{H}]^{+}$314.1387, found 314.1388.

methyl (4aS,7aS)-7-(hydroxymethyl)-1-oxo-2-(thiophen-2-ylmethyl)-2,4a,5,7a-tetrahydro-1 $\boldsymbol{H}$-cyclopenta[c]pyridine-4-carboxylate, $\mathbf{3 w}$. This compound was obtained as yellow oil in $69 \%$ yield; ${ }^{1} \mathrm{H}$ NMR $\left(400 \mathrm{MHz}, \mathrm{CDCl}_{3}\right) \delta 7.26(\mathrm{~s}, 1 \mathrm{H}), 7.24-7.17(\mathrm{~m}, 1 \mathrm{H}), 7.03-$ $6.96(\mathrm{~m}, 1 \mathrm{H}), 6.93(\mathrm{dtd}, J=5.1,3.1,1.5 \mathrm{~Hz}, 1 \mathrm{H}), 5.81(\mathrm{~s}, 1 \mathrm{H}), 5.00-4.73(\mathrm{~m}, 2 \mathrm{H}), 4.62$ $-4.39(\mathrm{~m}, 1 \mathrm{H}), 4.40-4.26(\mathrm{~m}, 2 \mathrm{H}), 3.81-3.63(\mathrm{~m}, 4 \mathrm{H}), 3.60-3.44(\mathrm{~m}, 1 \mathrm{H}), 2.93-$ $2.77(\mathrm{~m}, 1 \mathrm{H}), 2.30-2.12(\mathrm{~m}, 1 \mathrm{H}) .{ }^{13} \mathrm{C} \mathrm{NMR}\left(100 \mathrm{MHz}, \mathrm{CDCl}_{3}\right) \delta 170.3,166.6,141.0$ 138.1, 136.4, 129.4, 127.2, 127.0, 126.2, 112.0, 61.0, 51.7, 50.2, 45.6, 40.1, 37.5. HRMS (ESI) calcd for $\mathrm{C}_{16} \mathrm{H}_{18} \mathrm{NO}_{4} \mathrm{~S}[\mathrm{M}+\mathrm{H}]^{+}$320.0951, found 320.0957 . 
methyl

(4aS,7aS)-2-(4-fluorobenzyl)-7-(hydroxymethyl)-1-oxo-2,4a,5,7a-tetrahydro-1H-cycl openta[c]pyridine-4-carboxylate, 3x. This compound was obtained as white solid in $47 \%$ yield, $\mathrm{mp}=97-99{ }^{\circ} \mathrm{C} ;{ }^{1} \mathrm{H}$ NMR $\left(400 \mathrm{MHz}, \mathrm{CDCl}_{3}\right) \delta 7.25-7.17(\mathrm{~m}, 3 \mathrm{H}), 7.02(\mathrm{t}, J$ $=8.5 \mathrm{~Hz}, 2 \mathrm{H}), 5.84(\mathrm{~s}, 1 \mathrm{H}), 4.72(\mathrm{q}, J=14.9 \mathrm{~Hz}, 2 \mathrm{H}), 4.43-4.20(\mathrm{~m}, 2 \mathrm{H}), 3.79-3.67$ (m, 4H), 3.54 (dt, $J=10.9,8.7 \mathrm{~Hz}, 2 \mathrm{H}), 2.89$ (ddd, $J=18.2,8.7,2.4 \mathrm{~Hz}, 1 \mathrm{H}), 2.22$ (ddt, $J$ $=16.6,8.7,2.0 \mathrm{~Hz}, 1 \mathrm{H}) .{ }^{13} \mathrm{C} \mathrm{NMR}\left(100 \mathrm{MHz}, \mathrm{CDCl}_{3}\right) \delta 170.7,166.7,162.6(\mathrm{~d}, J=246.9$ Hz), 141.3, 136.8, 131.9 (d, $J=3.6 \mathrm{~Hz}), 129.7,129.6,129.5,116.1,115.9,112.0,61.1$, 51.8, 50.4, 50.2, 40.2, 37.5. HRMS (ESI) calcd for $\mathrm{C}_{18} \mathrm{H}_{19} \mathrm{FNO}_{4}[\mathrm{M}+\mathrm{H}]^{+}$332.1293, found 332.1294.

methyl (4aS,7aS)-7-(hydroxymethyl)-2-(4-methoxybenzyl)-1-oxo-2,4a,5,7a-tetrahydro-1H-c yclopenta $[c]$ pyridine-4-carboxylate, $3 \mathbf{y}$. This compound was obtained as yellow solid in $69 \%$ yield, $\mathrm{mp}=84-86{ }^{\circ} \mathrm{C} ;{ }^{1} \mathrm{H} \mathrm{NMR}\left(400 \mathrm{MHz}, \mathrm{CDCl}_{3}\right) \delta 7.21(\mathrm{~s}, 1 \mathrm{H}), 7.16(\mathrm{~d}, J=$ 8.7 Hz, 2H), $6.85(\mathrm{~d}, J=8.7 \mathrm{~Hz}, 2 \mathrm{H}), 5.91-5.74(\mathrm{~m}, 1 \mathrm{H}), 5.02(\mathrm{~s}, 1 \mathrm{H}), 4.79-4.52(\mathrm{~m}$, 2H), $4.42-4.23(\mathrm{~m}, 2 \mathrm{H}), 3.77(\mathrm{~s}, 3 \mathrm{H}), 3.74-3.66(\mathrm{~m}, 4 \mathrm{H}), 3.52(\mathrm{dt}, J=11.0,8.7 \mathrm{~Hz}$, 1H), $2.94-2.80(\mathrm{~m}, 1 \mathrm{H}), 2.21(\mathrm{ddt}, J=16.6,8.7,2.0 \mathrm{~Hz}, 1 \mathrm{H}) .{ }^{13} \mathrm{C} \mathrm{NMR}(100 \mathrm{MHz}$, $\left.\mathrm{CDCl}_{3}\right) \delta 170.7,166.7,159.5,141.1,136.9,129.6,129.2,128.1,114.4,111.7,61.0,55.4$ 51.7, 50.4, 50.2, 40.1, 37.6. HRMS (ESI) calcd for $\mathrm{C}_{19} \mathrm{H}_{22} \mathrm{NO}_{5}[\mathrm{M}+\mathrm{H}]^{+}$344.1492, found 344.1494 .

methyl (4aS,7aS)-7-(hydroxymethyl)-1-oxo-2-(1-phenylethyl)-2,4a,5,7a-tetrahydro-1H-cyclo penta[c]pyridine-4-carboxylate, 3z. This compound was obtained as colorless oil in 
45\% yield; ${ }^{1} \mathrm{H}$ NMR (400 MHz, $\left.\mathrm{CDCl}_{3}\right) \delta 7.40-7.26(\mathrm{~m}, 4 \mathrm{H}), 7.24-7.20(\mathrm{~m}, 1 \mathrm{H}), 7.10$ (s, 1H), 6.00 (p, $J=7.3 \mathrm{~Hz}, 1 \mathrm{H}), 5.84$ (ddt, $J=6.9,3.3,1.6 \mathrm{~Hz}, 1 \mathrm{H}), 4.44-4.27$ (m, 2H), $4.04(\mathrm{~s}, 1 \mathrm{H}), 3.80-3.64(\mathrm{~m}, 4 \mathrm{H}), 3.50(\mathrm{dtd}, J=11.0,8.6,4.8 \mathrm{~Hz}, 1 \mathrm{H}), 2.94-2.79(\mathrm{~m}$, 1H), $2.29-2.12(\mathrm{~m}, 1 \mathrm{H}), 1.58(\mathrm{~d}, J=7.2 \mathrm{~Hz}, 3 \mathrm{H}) .{ }^{13} \mathrm{C} \mathrm{NMR}\left(100 \mathrm{MHz}, \mathrm{CDCl}_{3}\right) \delta 170.3$, $166.8,141.3,139.4,133.4,129.5,129.0,128.2,127.1,112.0,61.1,51.7,51.4,50.5,40.1$, 37.1, 18.2. HRMS (ESI) calcd for $\mathrm{C}_{19} \mathrm{H}_{22} \mathrm{NO}_{4}[\mathrm{M}+\mathrm{H}]^{+} 328.1543$, found 328.1541 .

methyl

(4aS,7aS)-7-(hydroxymethyl)-1-oxo-2-(4-(trifluoromethyl)benzyl)-2,4a,5,7a-tetrahyd ro-1H-cyclopenta[c]pyridine-4-carboxylate, 3a'. This compound was obtained as colorless oil in $82 \%$ yield; ${ }^{1} \mathrm{H}$ NMR $\left(400 \mathrm{MHz}, \mathrm{CDCl}_{3}\right) \delta 7.53(\mathrm{~d}, J=8.0 \mathrm{~Hz}, 2 \mathrm{H}), 7.30$ (d, $J=8.0 \mathrm{~Hz}, 2 \mathrm{H}), 7.19(\mathrm{~s}, 1 \mathrm{H}), 5.79(\mathrm{~s}, 1 \mathrm{H}), 4.76(\mathrm{~s}, 2 \mathrm{H}), 4.37-4.22(\mathrm{~m}, 2 \mathrm{H}), 3.87-$ $3.69(\mathrm{~m}, 2 \mathrm{H}), 3.67(\mathrm{~s}, 3 \mathrm{H}), 3.52(\mathrm{dt}, J=10.9,8.7 \mathrm{~Hz}, 1 \mathrm{H}), 2.86(\mathrm{ddd}, J=16.8,8.6,2.7$ $\mathrm{Hz}, 1 \mathrm{H}), 2.26-2.13(\mathrm{~m}, 1 \mathrm{H}) .{ }^{13} \mathrm{C} \mathrm{NMR}\left(100 \mathrm{MHz}, \mathrm{CDCl}_{3}\right) \delta 170.4,166.5,141.3,140.1$, $136.7,130.0(\mathrm{q}, J=32.5 \mathrm{~Hz}), 128.8,127.7,125.8(\mathrm{q}, J=3.8 \mathrm{~Hz}), 123.9(\mathrm{q}, J=272.2 \mathrm{~Hz})$. 112.0, 60.8, 51.6, 50.2, 49.8, 40.0, 37.4. HRMS (ESI) calcd for $\mathrm{C}_{19} \mathrm{H}_{19} \mathrm{~F}_{3} \mathrm{NO}_{4}[\mathrm{M}+\mathrm{H}]^{+}$ 382.1261, found 382.1247.

\section{methyl}

(4aS,7aS)-7-(hydroxymethyl)-1-oxo-2-(pyridin-2-ylmethyl)-2,4a,5,7a-tetrahydro-1Hcyclopenta[c]pyridine-4-carboxylate, $3 \mathbf{b}^{\prime}$. This compound was obtained as white solid in $70 \%$ yield, $\mathrm{mp}=63-65{ }^{\circ} \mathrm{C} ;{ }^{1} \mathrm{H}$ NMR $\left(400 \mathrm{MHz}, \mathrm{CDCl}_{3}\right) \delta 8.65(\mathrm{~d}, J=4.8 \mathrm{~Hz}, 1 \mathrm{H})$, $7.86(\mathrm{t}, J=7.7 \mathrm{~Hz}, 1 \mathrm{H}), 7.47-7.36(\mathrm{~m}, 3 \mathrm{H}), 5.99(\mathrm{~s}, 2 \mathrm{H}), 5.85(\mathrm{~s}, 1 \mathrm{H}), 5.06-4.85(\mathrm{~m}$, $2 \mathrm{H}), 4.42-4.21(\mathrm{~m}, 2 \mathrm{H}), 3.79-3.71(\mathrm{~m}, 3 \mathrm{H}), 3.58(\mathrm{dt}, J=11.0,8.6 \mathrm{~Hz}, 1 \mathrm{H}), 2.99-2.85$ (m, 1H), 2.27 (ddt, $J=16.6,8.6,2.0 \mathrm{~Hz}, 1 \mathrm{H}) .{ }^{13} \mathrm{C} \mathrm{NMR}\left(100 \mathrm{MHz}, \mathrm{CDCl}_{3}\right) \delta 170.9$, 
166.6, 154.2, 147.6, 140.8, 139.4, 137.3, 129.8, 123.8, 123.6, 112.1, 60.9, 51.7, 51.3, 50.3, 40.0, 37.5. HRMS (ESI) calcd for $\mathrm{C}_{17} \mathrm{H}_{19} \mathrm{~N}_{2} \mathrm{O}_{4}[\mathrm{M}+\mathrm{H}]^{+}$315.1339, found 315.1342.

methyl

(4aS,7aS)-2-(3,4-dimethoxyphenyl)-7-(hydroxymethyl)-1-oxo-2,4a,5,7a-tetrahydro-1

$\boldsymbol{H}$-cyclopenta[c]pyridine-4-carboxylate, $\mathbf{3 c}$ '. This compound was obtained as yellow oil in $42 \%$ yield; ${ }^{1} \mathrm{H}$ NMR (400 MHz, $\left.\mathrm{CDCl}_{3}\right) \delta 7.37(\mathrm{~s}, 1 \mathrm{H}), 6.87(\mathrm{~d}, J=8.6 \mathrm{~Hz}, 1 \mathrm{H}), 6.76$ (dd, $J=8.5,2.4 \mathrm{~Hz}, 1 \mathrm{H}), 6.72(\mathrm{~d}, J=2.5 \mathrm{~Hz}, 1 \mathrm{H}), 6.07-5.90(\mathrm{~m}, 1 \mathrm{H}), 5.76(\mathrm{~s}, 1 \mathrm{H}), 5.29$ $-5.15(\mathrm{~m}, 2 \mathrm{H}), 3.87(\mathrm{~s}, 3 \mathrm{H}), 3.86(\mathrm{~s}, 3 \mathrm{H}), 3.85-3.80(\mathrm{~m}, 1 \mathrm{H}), 3.75(\mathrm{~s}, 3 \mathrm{H}), 3.66(\mathrm{dt}, J=$ 11.0, $8.7 \mathrm{~Hz}, 1 \mathrm{H}), 3.08-2.98(\mathrm{~m}, 1 \mathrm{H}), 2.37(\mathrm{ddt}, J=16.8,8.7,2.2 \mathrm{~Hz}, 1 \mathrm{H}) .{ }^{13} \mathrm{C}$ NMR $\left(100 \mathrm{MHz}, \mathrm{CDCl}_{3}\right) \delta 168.9,167.0,149.5,149.0,138.7,135.2,132.8,132.4,118.8,111.3$, 110.3, 110.2, 66.1, 56.2, 56.1, 51.9, 48.9, 40.3, 37.0. HRMS (ESI) calcd for $\mathrm{C}_{19} \mathrm{H}_{22} \mathrm{NO}_{6}$ $[\mathrm{M}+\mathrm{H}]^{+} 360.1442$, found 360.1444 .

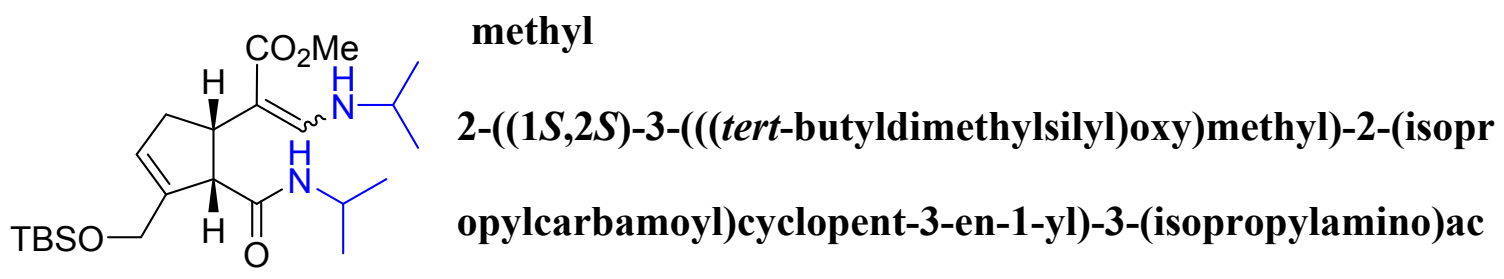
rylate, 3b-II. ${ }^{1} \mathrm{H}$ NMR $\left(400 \mathrm{MHz}, \mathrm{CDCl}_{3}\right) \delta 7.95-7.71(\mathrm{~m}, 1 \mathrm{H}), 6.78(\mathrm{~d}, J=13.1 \mathrm{~Hz}$, 1H), $5.86(\mathrm{~s}, 1 \mathrm{H}), 5.08(\mathrm{~d}, J=8.0 \mathrm{~Hz}, 1 \mathrm{H}), 4.20-4.06(\mathrm{~m}, 2 \mathrm{H}), 3.95-3.83(\mathrm{~m}, 1 \mathrm{H}), 3.69$ (s, 3H), $3.65-3.56(\mathrm{~m}, 1 \mathrm{H}), 3.39-3.23(\mathrm{~m}, 2 \mathrm{H}), 2.54-2.23(\mathrm{~m}, 2 \mathrm{H}), 1.15(\mathrm{t}, J=6.7 \mathrm{~Hz}$, $6 \mathrm{H}), 1.03(\mathrm{~d}, J=6.7 \mathrm{~Hz}, 3 \mathrm{H}), 0.94(\mathrm{~d}, J=6.5 \mathrm{~Hz}, 3 \mathrm{H}), 0.88(\mathrm{~s}, 9 \mathrm{H}), 0.04(\mathrm{~s}, 6 \mathrm{H}) .{ }^{13} \mathrm{C}$ NMR (100 MHz, $\left.\mathrm{CDCl}_{3}\right) \delta 170.83,170.80,149.7,143.1,128.1,90.9,62.1,56.0,50.5$, $49.9,42.1,40.9,35.4,26.1,24.2,24.1,23.01,22.95,18.5,-5.2,-5.3$. 